\title{
Inhibition of $T$ cell activation and autoimmune diabetes using a $B$ cell surface-linked CTLA-4 agonist
}

\author{
Brian T. Fife, ${ }^{1}$ Matthew D. Griffin, ${ }^{2}$ Abul K. Abbas, ${ }^{3}$ Richard M. Locksley, ${ }^{4}$ and Jeffrey A. Bluestone ${ }^{1}$ \\ 1UCSF Diabetes Center, Department of Medicine, UCSF, San Francisco, California, USA. ²Department of Internal Medicine, Division of Nephrology, \\ Mayo Clinic, Rochester, Minnesota, USA. ${ }^{3}$ Department of Pathology, ${ }^{4}$ Howard Hughes Medical Institute and \\ Departments of Medicine and Microbiology and Immunology, UCSF, San Francisco, California, USA.
}

\begin{abstract}
CTL-associated antigen 4 (CTLA-4) engagement negatively regulates $T$ cell activation and function and promotes immune tolerance. However, it has been difficult to explore the biology of selective engagement of CTLA-4 in vivo because CTLA-4 shares its ligands, B7-1 and B7-2, with CD28. To address this issue, we developed a Tg mouse expressing a single-chain, membrane-bound anti-CTLA-4 Ab (scFv) on B cells. B and T cells developed normally and exhibited normal phenotype in the steady state and after activation in these mice. However, B cells from $\mathrm{scFv} \mathrm{Tg}^{+}$mice $\left(\mathrm{sc} \alpha \mathrm{CTLA} 4^{+}\right)$prevented $\mathrm{T}$ cell proliferation and cytokine production in mixed lymphocyte reactions. Additionally, mice treated with sc $\alpha$ CTLA4 ${ }^{+}$B cells had decreased T celldependent $\mathrm{B}$ cell $\mathrm{Ab}$ production and class switching in vivo after antigen challenge. Furthermore, expression of this CTLA-4 agonist protected NOD mice from spontaneous autoimmune diabetes. Finally, this disease prevention occurred in Treg-deficient NOD.B7-1/B7-2 double-knockout mice, suggesting that the effect of the CTLA-4 agonist directly attenuates autoreactive $T$ cell activation, not Treg activation. Together, results from this study demonstrate that selective ligation of CTLA-4 attenuates in vivo $T$ cell responses, prevents development of autoimmunity, and represents a novel immunotherapeutic approach for the induction and maintenance of peripheral tolerance.
\end{abstract}

\section{Introduction}

CTL-associated antigen 4 (CTLA-4, also known as CD152) has been well established as a negative regulator of $\mathrm{T}$ cell function (1-3). CTLA-4 ligation antagonizes early $\mathrm{T}$ cell activation, leading to decreased IL-2 production, inhibition of cell cycle progression, decreased induction of cyclins by anti-CD3, and modulation of TCR signaling (2, 4-6). Mice deficient for CTLA-4 develop lymphoproliferative disease and die within 4 weeks of birth $(7,8)$ adding further evidence for the critical role of CTLA-4 inhibition of T cell responses. More recently, CTLA-4 has been implicated in the function of Tregs, which suppress effector $T$ cell activation and function $(9,10)$. In fact, it is clear that CTLA-4 plays a critical role in both the induction and maintenance of immune tolerance $(11,12)$. Thus, many efforts have focused on developing therapeutic approaches to selectively engage this pathway to treat autoimmunity and organ transplant rejection. Unfortunately, these attempts have been largely unsuccessful due to the complex biology of CTLA-4 (13). Early studies using Abs to target the CTLA-4 ligands B7-1 (also known as CD80) and B7-2 (also known as CD86) for immune regulation have been unreliable, as these ligands are shared with the positive costimulatory molecule CD28. Thus, rather than engaging CTLA-4 to turn off immunity, the ligands promote CD28/B7 interactions, resulting in enhanced $\mathrm{T}$ cell activation, expansion, and differentiation. Second, in vivo

Nonstandard abbreviations used: CTLA-4, CTL-associated antigen 4; DNP, dinitrophenyl; DP, double positive; KLH, keyhole limpet hemocyanin; mCTLA-4, mouse CTLA-4; MLR, mixed lymphocyte reaction; $\mathrm{P} \mu \mathrm{E} \mu$, IgM heavy-chain promoter and enhancer; sc $\alpha$ CTLA-4, single-chain anti-CTLA-4; scFv, single-chain antibody; SP, single positive; YFP, yellow fluorescent protein.

Conflict of interest: The authors have declared that no conflict of interest exists. Citation for this article: J. Clin. Invest. 116:2252-2261 (2006). doi:10.1172/JCI27856. administration of anti-CTLA-4 Abs routinely acts as an antagonist of CTLA-4 function. For instance, treatment of autoimmuneprone mice with anti-CTLA-4 results in the exacerbation of diseases, including experimental autoimmune encephalomyelitis and autoimmune diabetes $(14,15)$. Thus, a new approach to directly engage the CTLA-4 molecule with an agonist signal is essential for treating autoimmunity.

CTLA-4 signaling requires cross-linking in conjunction with $\mathrm{T}$ cell receptor engagement. This requirement is a direct consequence of the fact that CTLA-4 functions by directly influencing TCR-mediated signal transduction by altering TCR- $\zeta$ chain phosphorylation and immune synapse formation $(6,16,17)$. Thus, any effective negative signal following CTLA-4 engagement depends on the molecule being engaged, both physically and temporally, together with the TCR signal from the same APC (6). This most likely explains why in vivo treatment of mice with anti-CTLA-4 Abs acts as an antagonist, since even Fc receptor-mediated crosslinking would not likely occur with a cell bearing the relevant antigens. We hypothesized that effective delivery of a CTLA-4 agonist would depend on presentation of a specific CTLA-4 ligand (i.e., anti-CTLA-4 Ab) on a relevant APC-bearing cognate antigen. Thus, we developed a single-chain, membrane-bound anti-CTLA-4 $\mathrm{Ab}(\mathrm{scFv})$ that can be expressed directly on APCs. In previous studies, we demonstrated that this reagent was effective in vitro in blocking $\mathrm{T}$ cell activation and, as predicted, was effective when presented in cis, on the same APC, during TCR signaling (18). In this study, we have addressed the in vivo potential of such a reagent in an autoimmune setting. A construct was generated and expressed selectively on antigen-presenting B cells, which have been shown to be essential in secondary immunity and autoimmune diabetes in the NOD mouse strain (19). The model also takes advantage of the 

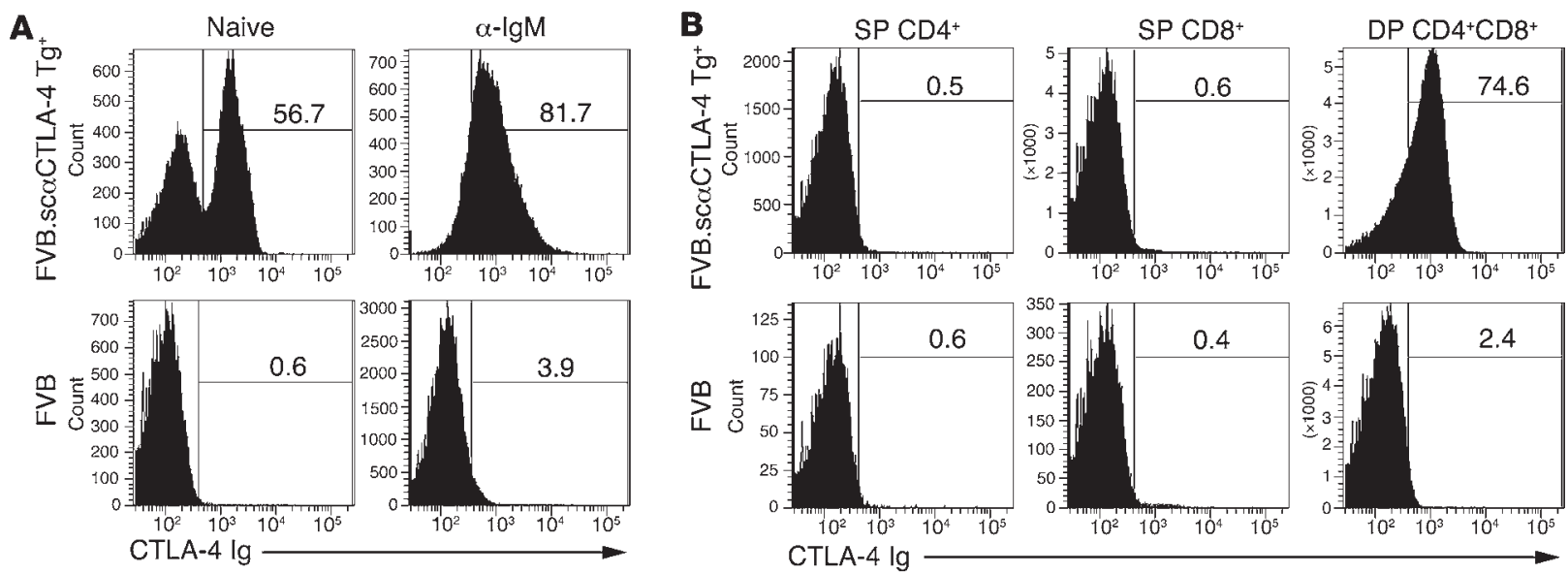

Figure 1

$\mathrm{Tg}^{+}$scaCTLA-4 expression. Flow cytometric analysis was performed on splenocytes from sc $\alpha \mathrm{CTLA}-4 \mathrm{Tg}^{+}$mice and littermate controls using soluble murine fusion protein mCTLA-4 Ig and anti-B220. (A) Upper panels demonstrate sc $\alpha$ CTLA-4 on naive $\mathrm{Tg}^{+}$B cells and activated Tg ${ }^{+}$ $B$ cells following anti-lgM stimulation (48 hours, $1 \mu \mathrm{g} / \mathrm{ml}$ ) compared with FVB littermate negative controls (lower panels). All plots were gated on B220+ B cells. (B) Flow cytometric analysis was performed on thymocytes from ScaCTLA-4 Tg mice and littermate controls as described above. Flow plots illustrate sc $\alpha$ CTLA-4 expression in DP CD4 ${ }^{+} C D 8^{+}$thymocytes from sc $\alpha C T L A-4 \mathrm{Tg}^{+}$mice, but not DP $\mathrm{CD} 4{ }^{+} \mathrm{CD} 8^{+}$thymocytes from FVB or SP CD4+ and SP CD8 ${ }^{+}$from sc $\alpha \mathrm{CTLA}-4 \mathrm{Tg}^{+}$or FVB Tg ${ }^{-}$controls. Results shown are representative of 3 independent experiments with $\geq 3$ mice per group.

motility of B cells that traffic to sites of antigen priming and participate in autoimmune reactions at sites of inflammation. Using this Tg mouse model, we demonstrate that cell surface-bound anti-CTLA-4 does not alter normal B or T cell development or function but selectively inhibits antigen-specific $T$ cell function in vivo and the development of autoimmune diabetes.

\section{Results}

Generation of membrane-bound anti-CTLA-4 Tg mice. The anti-CTLA-4 (clone 4F10) single-chain, membrane-bound (4F10scFv) construct has been well characterized in vitro and shown to have profound inhibitory effects on $\mathrm{T}$ cell function $(18,20)$. These results prompted us to develop a Tg mouse model to test this novel construct in vivo. We chose to express the molecule on $\mathrm{B}$ cells since previous studies showed that the ScFvmCTLA-4 was most effective when coexpressed on APCs, and B cells would be an effective cell population to selectively test antigen-specific responses. Thus, the IgM heavy-chain promoter and enhancer $(\mathrm{P} \mu \mathrm{E} \mu)$ was used to drive the expression of single-chain, anti-CTLA-4 (7M-4F10scFv) (21). In order to ensure that there would be no reverse signaling to the scFv-expressing cells, the construct was created using the transmembrane domain from $\mathrm{B} 7-1$, which lacked the $\mathrm{C}$ terminal intracytoplasmic tail $(18,20)$. The P $\mu$ E $\mu$ 7M-4F10scFv B7-1 construct was microinjected into day 1 embryos isolated from $\mathrm{FVB} / \mathrm{n}$ mice. B cell-specific 7M-4F10scFv B7-1 $\mathrm{Tg}^{+}$mice (hereafter referred to as scaCTLA-4) developed normally according to Mendelian fre-
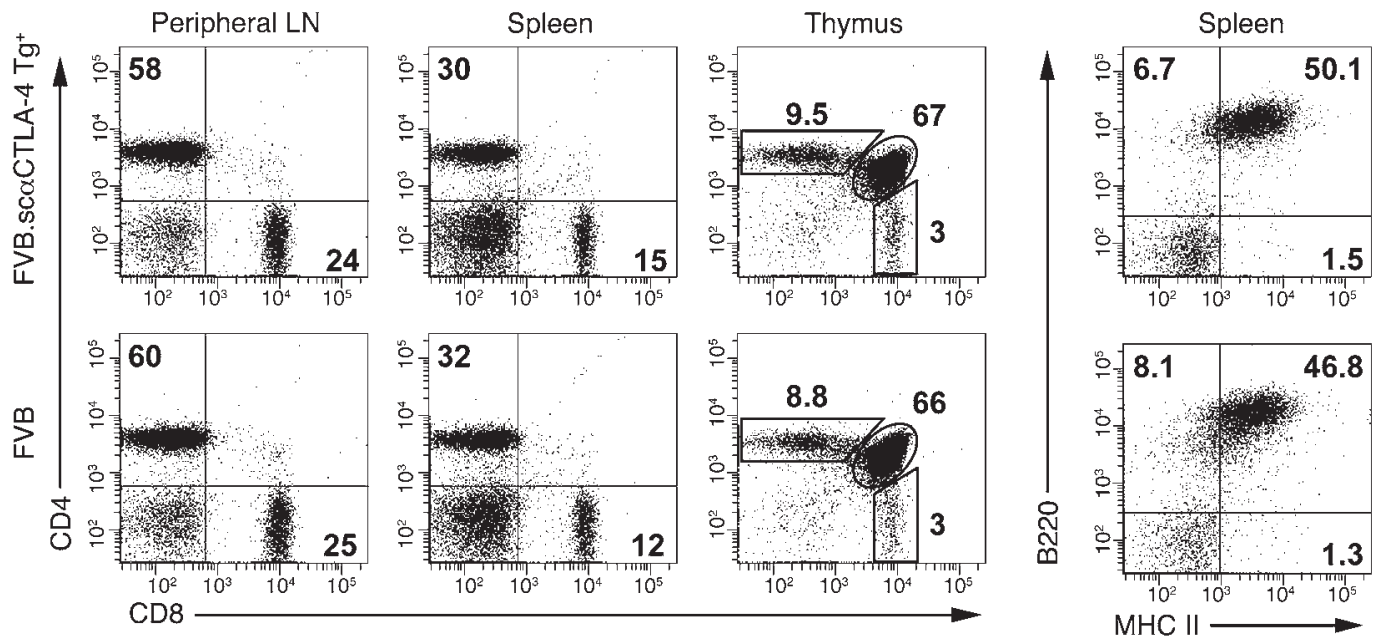

Figure 2

Characterization of $\mathrm{T}$ cells and B cells in scaCTLA-4 $\mathrm{Tg}^{+}$mice. Flow cytometric analysis was performed to determine the percentage of $\mathrm{CD} 4^{+} \mathrm{T}$ cells, CD8+ $\mathrm{T}$ cells, and B220+ B cells in the spleen, peripheral lymph nodes, and thymus from FVB and FVB.scaCTLA-4 Tg+ mice. The number in each histogram quadrant represents the percentage of total cells. Results shown are representative of 3 independent experiments with $\geq 3$ mice per group. 

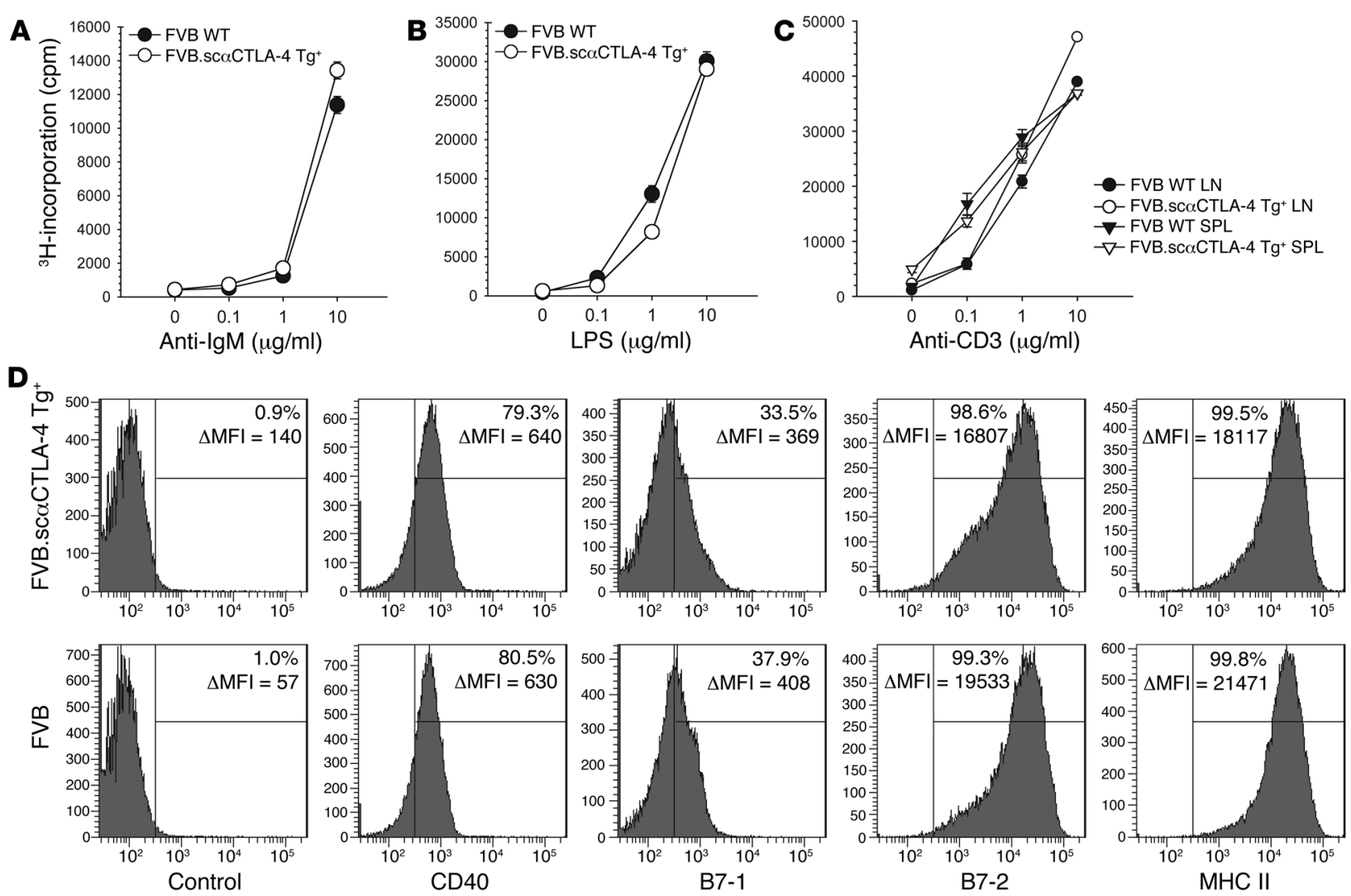

Figure 3

In vitro activation of $B$ cells and T cells from sc $\alpha C T L A-4 \mathrm{Tg}^{+}$mice. (A) B and T cell proliferation was assessed from scaCTLA-4 Tg ${ }^{+}$and controls using splenocytes following 0, 0.1, 1, or $10 \mu \mathrm{g} / \mathrm{ml}$ (A) anti-lgM, (B) LPS, or (C) anti-CD3 stimulation. (D) Flow cytometric analysis from scaCTLA-4 $\mathrm{Tg}^{+}$and FVB controls after 48-hour culture with anti-lgM $(1 \mu \mathrm{g} / \mathrm{ml})$. Shaded histogram represents positive stain for B7-1, B7-2, CD40, and MHC II. Percentage positive above isotype control with mean fluorescence intensity $(\Delta \mathrm{MFI})$ is shown. Histograms are gated on $\mathrm{B} 220^{+}$cells. Data presented are representative of 3 independent experiments with $\geq 3$ mice per group.

quencies and without gross defects. Tg scaCTLA-4 expression was identified by flow cytometry using murine CTLA-4 Ig. scaCTLA-4 expression was found on $B$ cells isolated from the spleen and peripheral lymph nodes. Approximately $55 \%$ of the B220+ B cells isolated from spleen of Tg mice were CTLA-4 Ig positive, with a range of $30 \%-60 \%$ (Figure $1 \mathrm{~A}$ ). This staining specificity was confirmed using human CTLA-4 Ig, which did not bind (data not shown). Stimulation of B cells with anti-IgM or LPS significantly increased the expression of scaCTLA- $4 \mathrm{Tg}^{+}$to more than $80 \%$ of the B220+ $\mathrm{B}$ cells (Figure 1A). scaCTLA-4 expression was also detected on $\mathrm{CD}^{+} \mathrm{CD}^{+}$double-positive (DP) thymocytes but not on single-positive (SP) $\mathrm{CD}^{+}$or $\mathrm{CD}^{+}$thymocytes (Figure $1 \mathrm{~B}$ ), peripheral $\mathrm{CD}^{+}$and $\mathrm{CD}^{+} \mathrm{T}$ cells, macrophages, or DCs (data not shown). It is also important to note that activated $\mathrm{CD} 4^{+}$and $\mathrm{CD} 8^{+}$ $\mathrm{T}$ cells did not express scaCTLA- 4 following anti-CD3 and antiCD28 activation (data not shown).

$T$ and $B$ cells develop normally in scaCTLA-4 $\mathrm{Tg}^{+}$mice. Despite scaCTLA-4 expression on DP thymocytes, T cell development appeared normal. This was evident in the thymus, with $70 \%$ $\mathrm{CD}^{+} \mathrm{CD}^{+} \mathrm{DP}, 9 \% \mathrm{CD}^{+} \mathrm{SP}$, and 3\% $\mathrm{CD}^{+}$SP thymocytes for both scaCTLA- $4 \mathrm{Tg}^{+}$and littermate controls (Figure 2). Moreover, we did not find any changes in the peripheral $\mathrm{T}$ cells from lymphocytes nor significant changes in the total spleen cellularity, based on total numbers or composition. Approximately 55\% B220+ B cells, 30\%
$\mathrm{CD}^{+} \mathrm{T}$ cells, and $15 \% \mathrm{CD}^{+} \mathrm{T}$ cells were observed in both groups (Figure 2). Additionally, we did not find any differences in marginal zone, follicular B cells, or transitional B cells in $\mathrm{Tg}^{+}$mice compared with controls (data not shown). $\mathrm{CD}^{+} \mathrm{T}$ cells constituted approximately $60 \%$ of the $\mathrm{T}$ cell composition of peripheral lymph nodes, with $25 \%$ being $\mathrm{CD}^{+} \mathrm{T}$ cells (Figure 2 ). These data indicated that although scaCTLA-4 expression was detected in the DP thymocytes, T and B cell development was normal.

scaCTLA-4 Tg $\mathrm{B}$ and $T$ cell function. The functional response of splenocytes (Figure 3, A and B) or purified B cells (data not shown) from scaCTLA-4 $\mathrm{Tg}^{+}$mice and littermate controls was compared using anti-IgM or LPS as mitogens. B cell proliferation, Ab production, IgM, MHC class II, CD40, B7-1, and B7-2 expression were measured. The expression of the $\mathrm{Tg}$ did not alter $\mathrm{B}$ cell responsiveness to BCR or innate stimuli (Figure $3, A$ and $B$ ). In addition, the surface expression of CD40, B7-1, B7-2, and MHC class II on naive and activated $\mathrm{Tg}^{+}$and $\mathrm{Tg}^{-} \mathrm{B}$ cells was comparable at 24,48 , and 72 hours (Figure 3D and data not shown).

We next assessed the functional potential of the peripheral $\mathrm{T}$ cell compartment. Purified T cells from spleen and peripheral lymph nodes were stimulated with plate-bound anti-CD3. Peripheral T cells isolated from scaCTLA- $4 \mathrm{Tg}^{+}$mice proliferated in a dosedependent response to TCR engagement in a manner similar to control FVB T cells (Figure 3C). CD69 upregulation and CD62L 

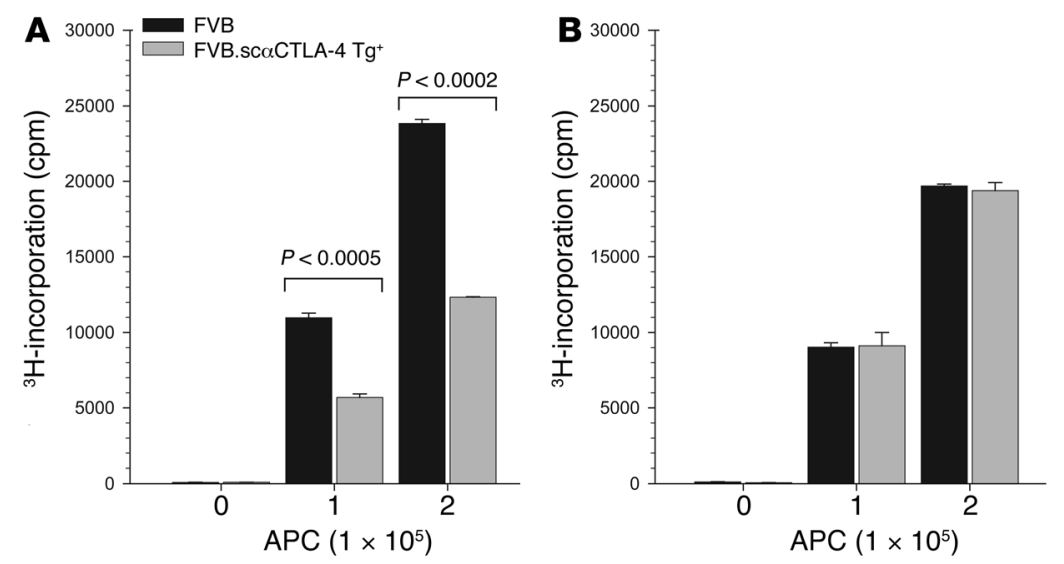

Figure 4

scaCTLA-4 Tg + B cells inhibit allogeneic T cell proliferation and cytokine production during a MLR T cell response. LPS-preactivated $B$ cells from FVB WT control or FVB.sc $\alpha$ CTLA-4 $\mathrm{Tg}^{+}$mice were incubated with purified (A) C57BL/6 or (B) C57BL/6.CTLA-4 $\mathrm{KO}$ responder $\mathrm{T}$ cells in a MLR. Proliferation was assessed by tritiated thymidine incorporation. (C and D) Culture supernatant from WT C57BL/6 responder $\mathrm{T}$ cells demonstrates that sc $\alpha \mathrm{CTLA}-4 \mathrm{Tg}^{+} \mathrm{B}$ cells significantly reduced (C) IFN- $\gamma$ and (D) IL-2 cytokine production after 48 hours of coculture. Responder $\mathrm{T}$ cells were cultured at $2 \times 10^{4}$ per well, and stimulator $B$ cells were cultured at either 0,1 , or $2 \times 10^{5}$ per well as indicated. Results illustrated are representative of at least 2 independent experiments.
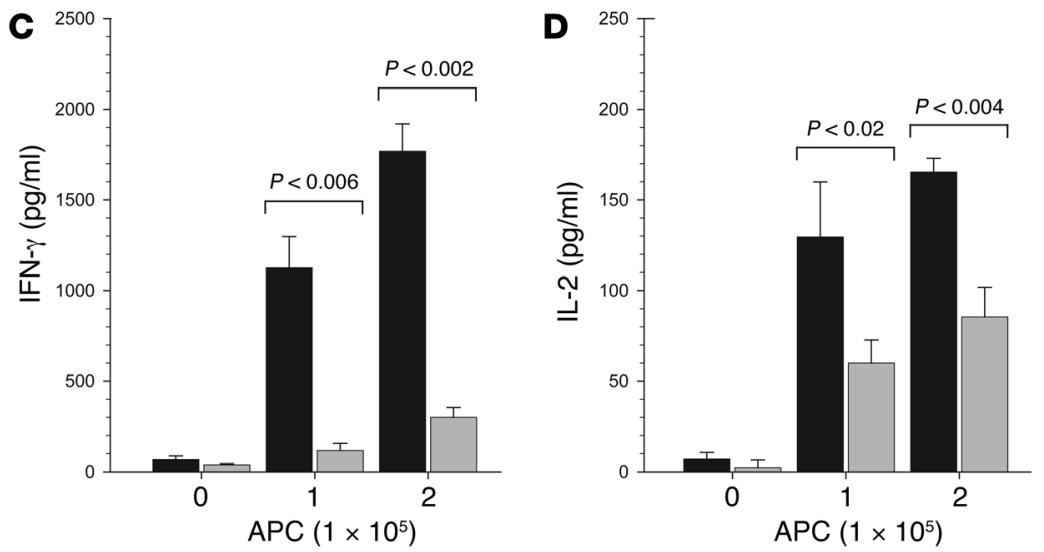

downmodulation were also normal upon activation in $\mathrm{Tg}^{+} \mathrm{T}$ and $\mathrm{B}$ cells (data not shown). Collectively, these data indicate that the $\mathrm{Tg}$ expression of scaCTLA-4 on B cells or DP thymic T cells did not significantly alter the development or function of peripheral $\mathrm{B}$ and $\mathrm{T}$ cells to polyclonal stimuli, including LPS, anti-IgM, or anti-CD3 and anti-CD28.

scaCTLA-4 Tg $\mathrm{T}$ cells inhibit $\mathrm{T}$ cell proliferation and cytokine production. Next, we examined whether the expression of scaCTLA-4 on allogeneic B cells would alter $\mathrm{T}$ cell activation in vitro in a mixed lymphocyte reaction (MLR). Responder C57BL/6 T cells were stimulated with MHC-mismatched purified CD $19^{+} \mathrm{B}$ cells from FVB scaCTLA- $4 \mathrm{Tg}^{+}$mice or littermate controls. $\mathrm{T}$ cell proliferation in response to scaCTLA- $4 \mathrm{Tg}^{+}$allogeneic $\mathrm{MHC}$ was significantly lower (>50\% reduction, $P<0.0002$ at $1: 5$ responder/stimulator ratio or higher) compared with control B cells (Figure 4A). Total splenocytes from FVB and FVB.scaCTLA-4 were not as effective (30\% reduction) at inhibiting $\mathrm{T}$ cell priming as purified $\mathrm{B}$ cells (50\%), suggesting endogenous DC activated the alloreactive $\mathrm{T}$ cells (data not shown). These data also suggest that scoCTLA-4 function did not inhibit in trans although the 30\% reduction in proliferation might suggest either a trans effect or, more likely, engagement of the activated $\mathrm{T}$ cells with antigen-bearing non- $\mathrm{Tg} \mathrm{B}$ cells or DCs. In this regard, stimulation with purified scaCTLA-4 $\mathrm{Tg}^{+} \mathrm{B}$ cells inhibited effector cytokine production in the allogeneic MLR. IFN- $\gamma$ production was suppressed by $83 \%(P<0.006)$ and IL-2 production by $68 \%(P<0.02)$ (Figure $4, C$ and D). IL-4 and IL-10 were undetectable, indicating there was not a switch to a Th2-type response (data not shown). The reduced response was dependent on CTLA-4 expression on responding T cells, as C57BL/6 T cells from CTLA-4 KO mice responded equally to scaCTLA- $4 \mathrm{Tg}^{+}$and $\mathrm{Tg}^{-} \mathrm{B}$ cell stimulations (Figure $4 \mathrm{~B}$ ). Thus, our results demonstrate that anti-CTLA-4 single-chain Abs expressed on APCs act as a CTLA-4 specific agonist to block responses of cognate T cells.

scaCTLA-4 inbibits T cell-dependent B cell Ab production in vivo. We next determined the effects of scaCTLA- 4 on immunological responses to hapten-carrier complexes in vivo. WT FVB mice received dinitrophenyl-OVA-pulsed (DNP-OVA-pulsed) B cells from either scaCTLA-4 $\mathrm{Tg}^{+}$mice or FVB controls (priming event). Seven days following initial priming, recipient mice received a boost of OVA antigen in CFA subcutaneously. DNP-specific Ab levels in sera were determined by using antigen-specific ELISA to determine levels of IgG1, IgG2a, and total Ig (days 0, 7, and 14). Mice immunized with antigen-pulsed B cells from the scaCTLA- $4 \mathrm{Tg}^{+}$mice showed significantly decreased DNP-specific Abs (Figure 5A, $P<0.0009)$. There was a particular suppression of class switching to IgG2a, suggesting that coengagement of CTLA-4 during antigen exposure led to a profound suppression of Th1-dependent $\mathrm{Ab}$ production. We did not detect significant differences in levels of IgG1 anti-DNP Abs, indicating that scoCTLA-4 did not cause a switch to a Th2 Ab response (Figure 5B) consistent with the in vitro cytokine responses. Furthermore, we did not detect a significant change in total Ig levels, indicating that the effects of scaCTLA-4 were limited to antigen-specific responses (Figure 5C). These results demonstrate the important role CTLA-4 has in regulating $\mathrm{T}$ cell activity using a novel Tg approach that directly engages CTLA- 4 on $T$ cells in vivo influencing both $\mathrm{T}$ cell and $T$ cell-dependent $B$ cell responses.

Effects of CTLA-4 in 2 models of autoimmune diabetes. The scaCTLA-4 $\mathrm{Tg}^{+}$mice were crossed onto the autoimmune diabetes-prone NOD 

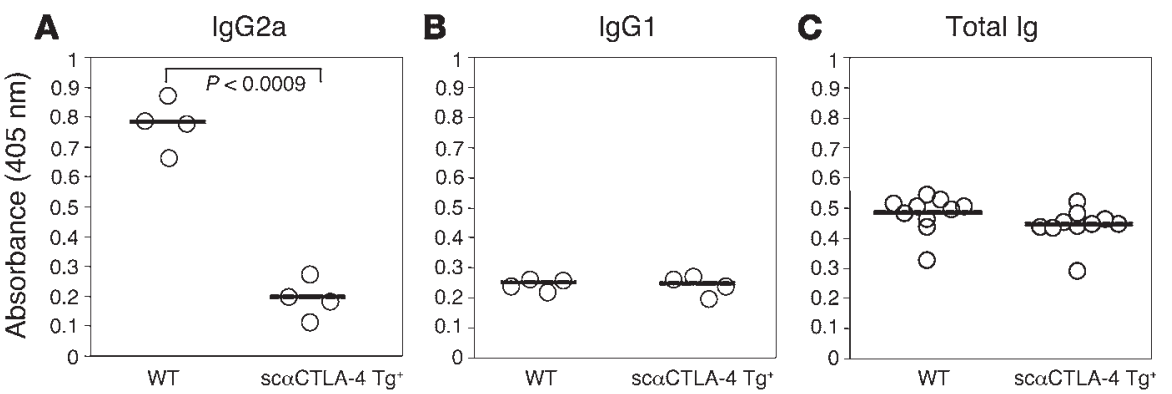

Figure 5

sc $\alpha$ CTLA-4 Tg ${ }^{+}$B cells inhibit T cell-dependent B cell IgG2a Ab production in vivo. Sc $\alpha$ CTLA-4 $\mathrm{Tg}$ and FVB littermate control B cells were activated in the presence of LPS $(1 \mu \mathrm{g} / \mathrm{ml})$ for 72 hours, loaded with DNP-OVA, and transferred to naive FVB recipients. Seven days following transfer of cells, recipient animals were boosted with $100 \mu \mathrm{g}$ DNP-OVA in CFA injected subcutaneously. Fourteen days following B cell transfer, anti-DNP Ab production was measured using DNP-KLH-specific ELISA. Shown in A, anti-DNP serum IgG2a from 4 individual WT or sc $\alpha$ CTLA-4 Tg+-treated mice. (B) Anti-DNP serum IgG1 from 4 individual WT or sc $\alpha$ CTLA-4 $\mathrm{Tg}^{+}-$treated mice. (C) Total Ig from 10 individual WT or scaCTLA-4 Tg+-treated mice. The mean for each group is shown with a horizontal line. Results shown are representative of at least 2 independent experiments.

mouse model to determine the effect of scaCTLA-4 B cell expression during the development of autoimmunity. Previous studies have shown that the development of diabetes in NOD mice is critically dependent on $\mathrm{B}$ cells but not $\mathrm{Ab}$ production, suggesting that $B$ cells are prominent APCs in this setting $(22,23)$. NOD mice develop spontaneous diabetes between 12 and 30 weeks of age, with approximately $70 \%$ disease incidence in female mice in our colony. FVB.sc $\alpha$ CTLA- $4 \mathrm{Tg}^{+}$mice were backcrossed 10 generations to the NOD background. NOD.sc $\alpha$ CTLA- $4 \mathrm{Tg}^{+}$mice were characterized as described for the FVB.sc $\alpha$ CTLA- $4 \mathrm{Tg}^{+}$mice for $\mathrm{B}$ and $\mathrm{T}$ cell development and function. We found similar expression of the $\mathrm{Tg}$ on resting and activated $\mathrm{B}$ cells and on DP thymocytes and no expression on resting or activated $\mathrm{CD} 4^{+}$or $\mathrm{CD} 8^{+}$peripheral $\mathrm{T}$ cells (data not shown). scaCTLA-4 Tg expression was not detected in cells isolated from the pancreas (data not shown). CD19+ purified scaCTLA-4+ $\mathrm{B}$ cells were also tested in the MLR assay as previously described and were found to inhibit allogeneic $\mathrm{T}$ cell responder C57BL/ 6 purified T cells in vitro (data not shown) similarly to the FVB.scaCTLA-4 $\mathrm{Tg}^{+}$cells (Figure 4). NOD.scaCTLA-4 and control NOD mice were monitored for the development of spontaneous diabetes. There was no statistical difference in insulin auto- $\mathrm{Ab}$ (IAA) responses in 5-, 10-, 20-, and 30-week-old NOD.scaCTLA-4 $\mathrm{Tg}^{+}$mice as compared with NOD littermates (data not shown). However, there was a significant reduction of spontaneous diabetes in NOD.scaCTLA- $4 \mathrm{Tg}^{+}$mice as compared with WT littermate NOD mice (Figure 6, $P<0.027$ ). NOD diabetes incidence reached $70 \%$ (12 of 17) by 30 weeks of age while only 33\% (5 of 15) of the NOD.scaCTLA- $4 \mathrm{Tg}^{+}$mice were diabetic by the end of the study period $(P<0.027)$. There was also a significant delay in disease kinetics and time to $25 \%$ diabetes incidence. NOD control mice were diabetic by 12.2 weeks while the same incidence frequency in the NOD.scaCTLA-4 $\mathrm{Tg}^{+}$mice did not occur until 19 weeks $(P<0.0077)$. Thus, expression of scaCTLA-4 Tg in NOD mice profoundly inhibited the development and progression of diabetes supporting a critical role for this pathway in autoimmunity.

Mechanism of CTLA-4-mediated inhibition: scaCTLA-4 inbibition in NOD.B7 double-knockout mice in the absence of Tregs. CTLA-4 has been pressed the development of diabetes

proposed to be involved in Treg function (9). CTLA-4 blockade has been shown to inhibit Treg suppression (9) and abrogate Treg-mediated protection of autoimmune colitis and transplant rejection $(24,25)$. To determine if the scaCTLA-4 $\mathrm{Tg}$ blocks diabetes by directly inhibiting autoreactive $T$ cells or affecting Treg activity, we took advantage of Tregdeficient NOD.B7-1.B7-2KO (NOD.B7 double-knockout [DKO]) mice. NOD.B7 DKO mice develop an accelerated form of autoimmune diabetes that begins as early as 2 weeks of age and progresses to 100 percent incidence (26). NOD. scaCTLA-4 $\mathrm{Tg}^{+}$mice were bred with B7-deficient NOD.B7 DKO mice and monitored for diabetes development. As previously reported, diabetes was exacerbated in the NOD.B7 DKO mice with $100 \%$ incidence at 17 weeks. In contrast, ectopic expression of scaCTLA-4 supby $40 \%$ (Figure 7A, $P<0.003$ ). Consistent with this finding, the $\mathrm{Tg}^{+}$scaCTLA-4 mice that were protected from diabetes also had significantly less insulitis (Figure 7B). Although Tregs have been shown to be deficient in NOD.B7 DKO mice (26), it remained possible that the engagement of CTLA-4 by the agonist scaCTLA-4 restored Tregs in the NOD.B7 DKO.scaCTLA- $4 \mathrm{Tg}^{+}$mice. We addressed this possibility by FACS analysis and determined that Tregs were not restored in NOD.B7 DKO.scaCTLA- $4 \mathrm{Tg}^{+}$mice. Both B7 DKO groups had $0.3 \% \mathrm{CD} 4{ }^{+} \mathrm{CD} 25^{\text {hi }} \mathrm{CD} 62 \mathrm{~L}^{+}$Tregs in comparison with $4 \% \mathrm{CD} 4{ }^{+} \mathrm{CD} 25^{\text {hi }} \mathrm{CD} 62 \mathrm{~L}^{+}$Tregs in WT NOD mice (Figure 7C). Recent studies suggest that the putative transcription factor FoxP3 is the best marker of mouse Tregs. Therefore, we repeated the analysis of $\mathrm{B} 7 \mathrm{DKO}$ mice, utilizing intracellular stain-

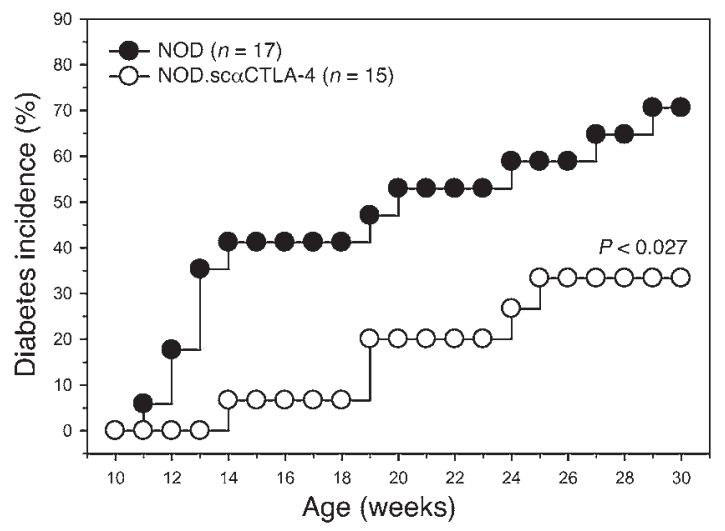

Figure 6

NOD.sc $\alpha$ CTLA- $4 \mathrm{Tg}^{+}$mice are protected from spontaneous diabetes. Female NOD.sc $\alpha$ CTLA-4 $\mathrm{Tg}^{+}$mice and NOD littermate controls were monitored for the development of spontaneous autoimmune diabetes. Diabetes incidence is shown here for NOD $(n=17)$ (filled circles) and NOD.sc $\alpha C T L A-4 \operatorname{Tg}^{+}(n=15)$ (open circles). NOD diabetes incidence reached $70 \%$ (12 of 17) by 30 weeks of age while diabetes incidence in NOD.sc $\alpha$ CTLA- $4 \mathrm{Tg}^{+}$mice was significantly reduced to $33 \%$ (5 of $15)$ by 30 weeks of age $(P<0.027)$. 


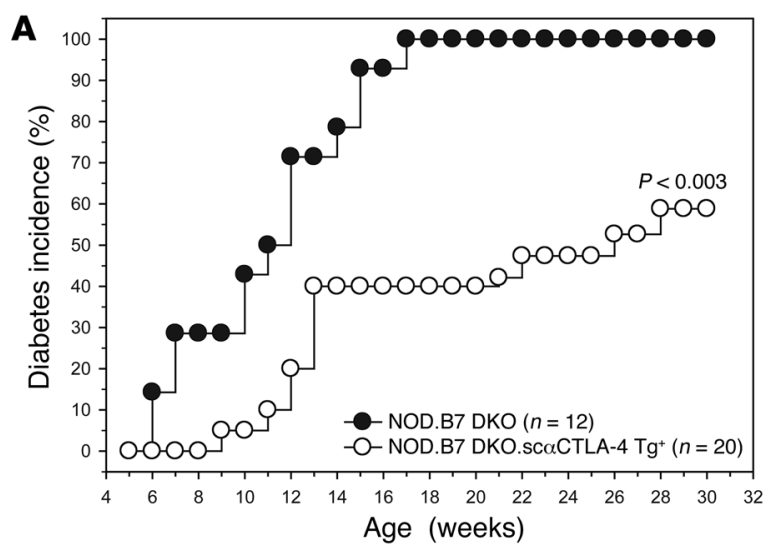

B
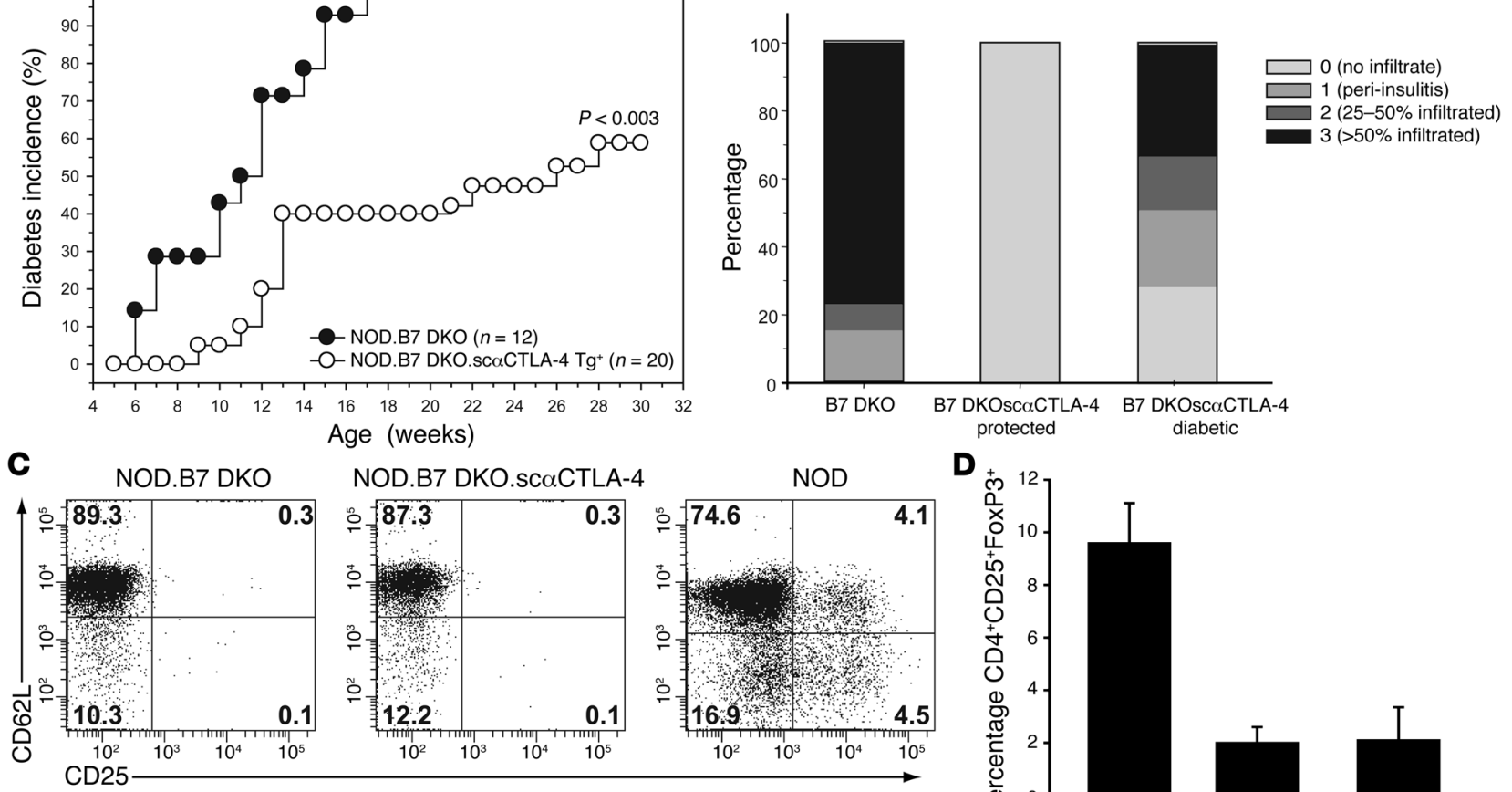

Figure 7

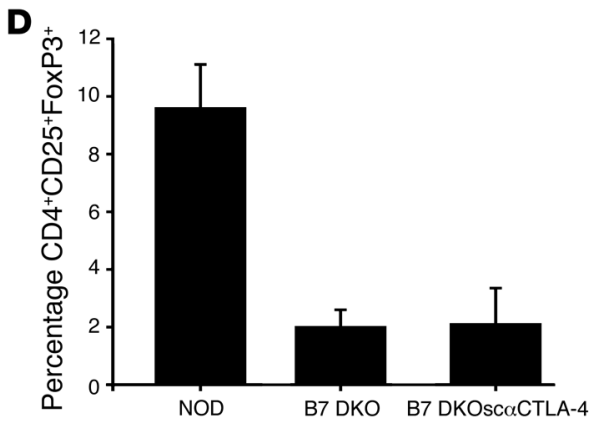

scaCTLA-4 Tg+ NOD.B7 DKO mice are protected from accelerated diabetes. Female NOD.B7 DKO and NOD.B7 DKO.sc $\alpha$ CTLA-4 Tg ${ }^{+}$mice were monitored for the development of spontaneous disease. (A) Diabetes incidence is shown here for NOD.B7 DKO ( $n=12$ ) (open circles) and NOD.B7 DKO.sc $\alpha$ CTLA-4 Tg ${ }^{+}$mice $(n=20)$ (filled circles). NOD.B7 DKO diabetes incidence reached $100 \%$ while diabetes incidence in NOD.B7 DKO.sc $\alpha$ CTLA-4 Tg+ mice was significantly reduced to $60 \%$ by 30 weeks of age $(P<0.003)$. (B) NOD.B7 DKO.sc $\alpha C T L A-4$ Tg ${ }^{+}$mice have decreased insulitis at 14 weeks of age. Pancreas from 14-week-old NOD.B7 DKO and scaCTLA-4 Tg NOD.B7 DKO mice were stained with H\&E to determine clinical severity of insulitis. Pancreatic islets were scored for the presence of mononuclear infiltration: 0 , no infiltrate; 1 , peri-insulitis present; $2,25-50 \%$ of the islet contained infiltrate; 3 , more than $50 \%$ of the islet was infiltrated. Average percentages shown were determined from at least 100 islets from at least 3 mice per group. (C) Flow cytometric analysis was performed to determine the percentage of $\mathrm{CD}^{+}{ }^{+} \mathrm{CD} 25^{+} \mathrm{CD} 62 \mathrm{~L}^{+}$and (D) CD4+CD25+FoxP3+ ${ }^{+}$Tregs in the lymphoid tissue of NOD.B7 DKO, NOD. B7 DKO.scaCTLA-4 Tg+, and NOD mice. The mean \pm SD from at least 3 mice per group is shown. Results shown are representative of 3 independent experiments.

ing of FoxP3 on $\mathrm{CD}^{+} \mathrm{CD} 25^{+}$Tregs. As seen in Figure 7D, there is a significant decrease in the percentage of $\mathrm{CD} 4^{+} \mathrm{CD} 25^{+} \mathrm{FoxP} 3^{+}$ Tregs in the NOD.B7 DKO mice as compared with the NOD mice $(P<0.0002)$. This defect in Treg numbers was not reconstituted in NOD.B7 DKO.scaCTLA4 $\operatorname{Tg}^{+}(P<0.0004)$. Furthermore, there was no statistical difference in the number of FoxP3 ${ }^{+} \mathrm{T}$ cells between NOD.B7 DKO and NOD.B7 DKO.scaCTLA-4 $\mathrm{Tg}^{+}$mice $(P<0.95)$. These results suggest that the inhibition of disease by sc $\alpha$ CTLA- 4 was not due to a restoration of Tregs; rather, the scaCTLA-4 Tg may directly inhibit autoreactive $\mathrm{T}$ cells.

scaCTLA-4 Tg $\mathrm{g}^{+}$B cells inhibit the activation of autoreactive $T$ cells in pancreatic lymph nodes. To further characterize $\mathrm{T}$ cell inhibition and test the hypothesis that CTLA-4 engagement directly inhibits autoreactive $\mathrm{T}$ cells, we compared islet antigen-specific $\mathrm{T}$ cell priming in the pancreatic LN of NOD.B7 DKO versus NOD. B7 DKO.scaCTLA-4 $\mathrm{Tg}^{+}$recipients. Islet antigen-specific TCR $\mathrm{Tg}^{+}$BDC2.5 Thy 1.1 congenic $\mathrm{CD} 4{ }^{+} \mathrm{CD} 25^{-} \mathrm{CD} 62 \mathrm{~L}^{+} \mathrm{LN}$ cells were labeled with CFSE and adoptively transferred into prediabetic NOD.B7 DKO or NOD.B7 DKO.scaCTLA-4 $\mathrm{Tg}^{+}$mice, and their proliferation was measured by CFSE dilution as a readout of in vivo priming (27). Antigen-specific Tregs were depleted to address the role of scaCTLA- 4 on autoreactive effector T cells in the absence of regulation (9). As seen in Figure 8, 70.7\% (65.3\% $\pm 4.7 \%)$ of the autoreactive BDC2.5 cells went into cycle in the pancreatic LN from NOD.B7 DKO recipient mice compared with only $22.1 \%(28.8 \% \pm 10.7 \%)$ in NOD.B7 DKO.scaCTLA- $4 \mathrm{Tg}^{+}$recipients (Figure 8A). Moreover, BDC2.5 CD $4^{+} \mathrm{T}$ cells divided only 3 generations in NOD.B7 DKO.scaCTLA-4 hosts in contrast to 6 to 8 generations in the NOD.B7 DKO recipients (Figure 8A). There was no proliferation observed in antigen-deficient inguinal $\mathrm{LN}$ in either NOD.B7 DKO or NOD.B7 DKO.scaCTLA-4 Tg+ mice (Figure $8 \mathrm{~A})$. These results indicate that $\mathrm{B}$ cells bearing surface-bound anti-CTLA-4 suppress in vivo priming of autoreactive T cells in the absence of Tregs.

Next, we determined whether scaCTLA-4 similarly affects the ability of autoreactive BDC2.5 CD4 ${ }^{+} \mathrm{T}$ cells to differentiate and produce Th1 effector cytokines such as IFN- $\gamma$, which is known to be involved in disease pathogenesis (28). For this study, we utilized an IFN- $\gamma$ reporter mouse in which the expression of yellow fluorescent protein (YFP) is under the control of the IFN- $\gamma$ promoter (29). These targeted reporter mice were designated Yeti, for yellow-enhanced transcript for IFN- $\gamma$. Direct assessment of in vivo differentiation and IFN- $\gamma$ production can thus be measured by flow cytometry ex vivo without the requirement of in vitro restimulation. We backcrossed these mice 12 generations to NOD and subsequently bred to NOD.BDC2.5.Thy1.1 TCR Tg 


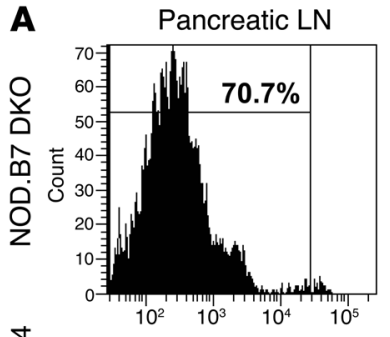

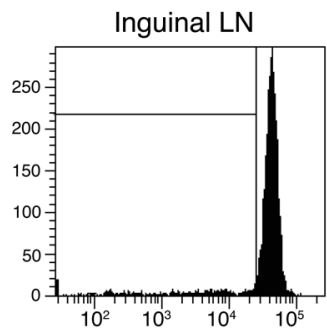

B Pancreatic LN
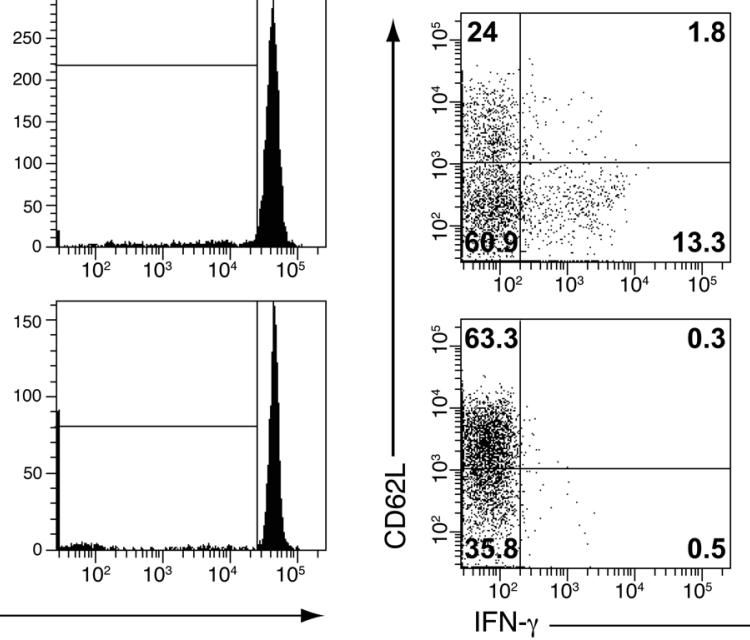

Inguinal LN
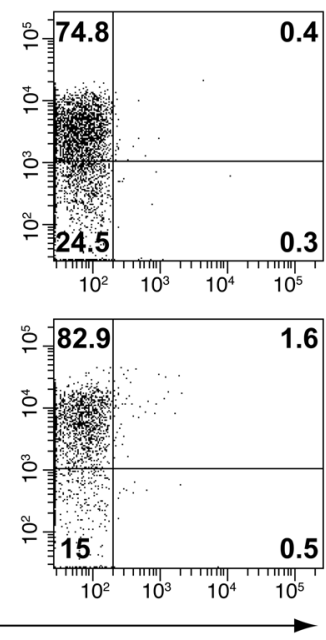

\section{Figure 8}

scaCTLA-4 Tg+ B cells inhibit the activation of autoreactive T cells in pancreatic lymph nodes. BDC2.5. Thy1.1 CD4+ LN cells were labeled with CFSE and adoptively transferred to prediabetic 6-week-old NOD.B7 DKO or NOD.B7 DKO.scaCTLA-4 Tg mice. Pancreatic and inguinal LN cells were harvested from recipients ( $n=3$ for each group) 5 days later for analysis by flow cytometry for CFSE dye dilution as a readout of in vivo priming and proliferation. (A) Representative histogram CFSE plots of CD4+CD90.1+ T cells from NOD.B7 DKO and NOD.B7 DKO.sc $\alpha$ CTLA-4 Tg ${ }^{+}$recipients. (B) Direct assessment of in vivo differentiation and IFN- $\gamma$ production measured by flow cytometry ex vivo. CD4 ${ }^{+}$CD25- T cells from BDC2.5. Thy1.1. Yeti mice transferred to prediabetic NOD.B7 DKO or NOD.B7 DKO.sc $\alpha$ CTLA-4 recipients $(n=3$ for each group). Shown here is YFP (IFN- $\gamma$ ) and CD62L expression from CD4+CD90.1 ${ }^{+} \mathrm{T}$ cells from pancreatic and inguinal $\mathrm{LNs}$. Results shown are representative of 2 independent experiments.

mice to derive NOD.BDC2.5.Thy1.1.Yeti mice. The CD4+ $\mathrm{T}$ cells from BDC2.5.Thy1.1.Yeti mice were adoptively transferred to prediabetic NOD.B7 DKO or NOD.B7 DKO.scaCTLA-4 $4^{+}$recipients, and the number of YFP-expressing $\mathrm{T}$ cells in the pancreatic and inguinal LN were determined by FACS. It is important to note that freshly isolated $\mathrm{CD} 4^{+} \mathrm{T}$ cells from NOD.BDC2.5.Thy1.1.Yeti mice did not express YFP (data not shown). scaCTLA- $4 \mathrm{Tg}^{+} \mathrm{B}$ cells blocked the IFN $-\gamma$ production in the pancreatic LN $0.47 \% \pm 0.06 \%$ compared with NOD.B7 DKO recipients with $10.3 \% \pm 3 \%$ IFN- $\gamma$ YFP-positive cells (Figure 8B). CD62L expression was also measured as a parameter of in vivo priming of $\mathrm{CD} 4^{+} \mathrm{BDC} 2.5$.Thy $1.1^{+}$ gated cells from pancreatic and inguinal LN. IFN- $\gamma$-positive cells from the NOD.B7 DKO recipients downmodulated CD62L further, indicating in vivo activation in the absence of CTLA-4 signal. BDC2.5 cells from NOD.B7 DKO.scaCTLA-4 recipient mice did not express YFP, and only $35.8 \%(34.7 \% \pm 1.4 \%)$ of the $\mathrm{CD}^{+}$ cells from pancreatic LN downmodulated CD62L compared with $60.9 \%(59.9 \% \pm 2.3 \%)$ in controls (Figure $8 \mathrm{~B})$. The vast majority of $\mathrm{CD}^{+}$BDC2.5.Thy1.1 cells $(\sim 75 \%)$ from the inguinal $\mathrm{LN}$ in both groups did not express IFN- $\gamma$ or downmodulate CD62L, indicating a lack of $\mathrm{T}$ cell priming as predicted (Figure $8 \mathrm{~B}$ ). Taken together, the results demonstrate that B cells expressing scaCTLA- 4 can efficiently suppress proliferation and differentiation of autoreactive $\mathrm{T}$ cells in the draining $\mathrm{LN}$ in vivo (30).

\section{Discussion}

Although CTLA-4 engagement by B7 has been demonstrated to downregulate $\mathrm{T}$ cell responses in several systems, lack of appropriate reagents has prevented direct ligation in vivo to inhibit $\mathrm{T}$ cell function in autoimmune diseases. Rather, the majority of studies have utilized CTLA-4 antagonists or ectopic expression of B7 (which stimulate both CD28 and CTLA-4) to implicate the role of CTLA-4 in vivo (refs 14, 15 and reviewed in refs. 13, 31). In the studies presented here, we have generated a model to examine the effect of selective engagement of CTLA-4 during in vivo immune responses. This was accomplished by expressing a single-chain, membrane-bound $\mathrm{scFv}$ anti-CTLA-4 selectively on B cells. Our results demonstrate that sc $\alpha$ CTLA- $4 \mathrm{Tg}^{+}$expression did not affect $\mathrm{T}$ or $\mathrm{B}$ cell development but profoundly inhibited $\mathrm{T}$ cell responses to antigens presented by scaCTLA- $4 \mathrm{Tg}^{+} \mathrm{B}$ cells in vitro and in vivo. Moreover, we show that selective engagement of CTLA- 4 conferred significant protection against disease in 2 models of autoimmune diabetes, even in the absence of Tregs. The mechanism for this regulation was determined to be the prevention of autoreactive $T$ cell expansion and development of effector function.

CTLA-4 has previously been demonstrated to regulate peripheral tolerance in several experimental systems $(11,12,32)$. This regulation is at the level of $\mathrm{T}$ cell expansion and differentiation. In particular, we have previously shown that selective engagement of CTLA-4 using membrane-bound scaCTLA-4 inhibits antigenspecific $\mathrm{T}$ cell responses in vitro $(18,20)$. We have also shown that scaCTLA- 4 can regulate $\mathrm{CD} 8^{+} \mathrm{T}$ cell-mediated tumor clearance in an in vivo tumor model (33). $\mathrm{CD}^{+} \mathrm{T}$ cells isolated from animals bearing scaCTLA-4 tumor cells were hyporesponsive ex vivo upon antigen rechallenge, indicating that CTLA- 4 engagement resulted in tolerance (33). In this study, we have extended our previous findings to show that scaCTLA- 4 expression on B cells inhibits $\mathrm{CD}^{+}$autoimmune responses in vivo. Two separate experimental models of autoimmune diabetes were used to address the efficacy of membrane-bound anti-CTLA-4 for disease prevention. We demonstrate that selective engagement of CTLA- 4 conferred significant protection against disease in spontaneous NOD diabetes and even in the more aggressive autoimmune NOD.B7-1/B7-2 KO (B7 DKO) model. This CTLA-4 control may function at several 
levels, including $\mathrm{T}$ cell activation/proliferation, Th1/Th2 differentiation, and cell trafficking. Our data suggest that scoCTLA-4mediated inhibition is at the level of $\mathrm{CD} 4^{+} \mathrm{T}$ cell activation/differentiation. We found a significant decrease in the percentage of autoreactive BDC2.5 $\mathrm{CD}^{+} \mathrm{T}$ cells proliferating in the NOD.B7 DKO.sc $\alpha$ CTLA- $4 \mathrm{Tg}^{+}$in pancreatic LN compared with controls, indicating that CTLA-4 engagement had a direct effect on antigen-specific $\mathrm{T}$ cell activation and expansion. $\mathrm{CD} 8^{+} \mathrm{NRP}-\mathrm{V} 7^{+}$tetramer-positive cells were examined in the NOD.scaCTLA- $4 \mathrm{Tg}^{+}$ mice, as these T cells have been shown to be increased during the progression of diabetes in NOD mice (34). There were no statistical differences in the number of $\mathrm{CD}^{+} \mathrm{NRP}-\mathrm{V} 7^{+}$antigen-specific cells in the NOD.scaCTLA- $4 \mathrm{Tg}^{+}$mice as compared with NOD mice (data not shown). We further demonstrated scaCTLA-4 significantly limited the differentiation of diabetogenic T cells toward Th1 IFN- $\gamma$-producing cells. NOD.B7 DKO.scaCTLA- $4 \mathrm{Tg}^{+}$ mice had significant reduction of IFN- $\gamma / \mathrm{YFP}^{+}$cells $(0.5 \%)$ compared with controls $(13.3 \%)$ in islet-draining pancreatic LN. These data indicate that CTLA-4 engagement significantly inhibits both the proliferation and Th1 differentiation in an antigen-specific response. The protection from disease in this setting appears to be a result of direct inhibition of effector $\mathrm{T}$ cell activation/differentiation rather than regulation, as the Treg deficiency observed in NOD.B7 DKO mice was not restored by the expression of the scaCTLA-4 Tg. It is interesting to note that reduced disease incidence correlated with decreased tissue infiltration (Figure 7B). This is not surprising unless you consider the fact that nearly all female NOD mice develop insulitis by 3 to 4 weeks of age (35). This lack of pancreatic infiltration indicates that CTLA-4 may function to prevent the trafficking and accumulation of inflammatory infiltrates, likely at the level of LN priming. It is important to note that the presence of the scaCTLA-4 Tg did not render these mice immunocompromised, as NOD.sc $\alpha$ CTLA- $4 \mathrm{Tg}^{+}$mice cleared cytomegalovirus in a manner similar to that observed in NOD mice. In these experiments, NOD.RAG KO mice were used to demonstrate that immunocompromised animals were unable to clear this viral infection (data not shown). Therefore, the scaCTLA-4-mediated disease inhibition and decreased insulitis in the absence of Tregs support the interpretation that CTLA-4 ligation functioned directly on the autoreactive $T$ cells. These data are consistent with previous findings that CTLA-4 engagement results in a tolerant state, and only after blocking CTLA-4 do tissue infiltration and autoimmunity occur.

scaCTLA-4 $\mathrm{Tg}^{+} \mathrm{B}$ cells prevent destructive insulitis and significantly affect NOD and NOD.B7 DKO mice from developing diabetes, suggesting $\mathrm{B}$ cells play a pivotal role in disease progression. B cells have been shown to be critical for the development of autoimmune diabetes in NOD mice, as B cell-deficient mice are protected $(22,23)$. Interestingly, B cell-deficient mice develop some insulitis, suggesting that diabetogenic responses can be initiated in the absence of B cells, likely via DCs. However, diabetes fails to progress in these mice, demonstrating a critical role of $B$ cells in the amplification of autoimmune responses and disease progression (22). Wong et al. recently demonstrated that B cell antigen presentation, not Ig production, is essential for development of insulitis and diabetes (23). In fact, B cells may contribute to disease pathogenesis by further activating $T$ cells in the pancreas or by enhancing epitope spreading where islet $\mathrm{Ab}$-producing $B$ cells selectively process apoptotic $\beta$ cells to present new islet epitopes. Falcone et al. demonstrated a critical role for B cell antigen presentation and development of spontaneous responses to self antigens during the progression of autoimmune diabetes (19). Thus, it is possible that in our model, scaCTLA- $4 \mathrm{Tg}^{+}$expression controlled diabetes by preventing B cell-dependent amplification of the anti-islet responses.

scaCTLA-4 significantly delayed disease onset and development of spontaneous diabetes in NOD mice. Although there was not complete protection, there is a significant consequence of engaging CTLA-4 during development of autoimmune diabetes. There are a number of possibilities as to why $B$ cell expression of anti-CTLA-4 was not sufficient for complete disease protection. First, autoreactive $\mathrm{T}$ cells may interact with several APCs during diabetes initiation. Tg B cell expression of anti-CTLA-4 may raise the threshold for $\mathrm{T}$ cell activation. However, it is possible that some autoreactive T cells escape scaCTLA-4 regulation and encounter islet antigens presented by DCs or macrophage cells in the absence of CTLA-4. This possibility is supported by an in vitro MLR assay where total splenocytes were used to stimulate MHC mismatched T cells. In this assay, the presence of endogenous Tg nonbearing APCs partially restored $\mathrm{T}$ cell proliferation. These data further support the notion that the anti-CTLA-4 is working directly on $\mathrm{T}$ effectors and not indirectly through Tregs. A second possibility is that CTLA-4 may function to skew the T cell repertoire. However, based on previous studies by Allison and colleagues, it would be expected that, if anything, the repertoire would be broadened to include lower affinity clones that would escape CTLA-4 inhibition due to decreased action of the inhibitory receptor (36). A third possibility is that, over time, CTLA-4resistant $\mathrm{T}$ cell clones emerge by overcoming the threshold of CTLA-4-mediated inhibition and become less dependent on costimulation. Autoimmune diabetes is a chronic progressive disease with autoreactive $\mathrm{T}$ cells accumulating within the target organ months prior to clinical manifestations (35). Given the disease kinetics, it is possible that autoreactive cells may develop mechanisms to overcome or bypass CTLA-4-mediated suppression. This idea is supported by the work of You et al., where equal frequencies of diabetogenic T cells were detected in 6-week-old prediabetic and overtly diabetic NOD mice (37). They proposed that the progression to overt disease correlates with a qualitative change in the pathogenic $T$ cells that allows them to escape from regulation over time (37). Thus, in a chronic progressive autoimmune disease, such as type I diabetes, evolution of pathogenic $\mathrm{T}$ cells is likely to post significant challenge in designing novel immunosuppressive therapies.

Overall, we have demonstrated anti-CTLA-4 Abs anchored on the surface of APCs to be an effective means to suppress autoimmune responses. There are a number of ways that can be envisioned to adapt such an approach in clinical settings to prevent graft rejection and treat autoimmunity. The first is a gene therapy approach targeting APCs, including macrophages, DCs, and B cells, to express this construct with the intent that antigen-specific responses would be selectively engaged together with CLTA-4 inhibition. A second approach shown to be effective for targeting autoimmunity was the use of a bispecific $A b$ to simultaneously engage antigen and CTLA-4 (38). This approach was effective in a mouse model of thyroiditis using bispecific Abs against thyroid-stimulating hormone receptor and CTLA-4 (39). The major limitation for this strategy is the requirement of identifying and generating tissue-specific Abs against immunodominant antigens for each disease. The last approach to selectively target autoreactive and 
alloreactive T cells is to modify the membrane of APCs or grafted tissue with inhibitory molecules. Recent reports have demonstrated effective use of cell membrane-anchored FasL to prevent islet and cardiac graft rejection (40). This approach utilizes a protocol to biotinylate cells and "decorate" the membrane with a chimeric protein comprised of streptavidin and FasL (40). This is an attractive approach for the clinical setting for treatment of autoimmune disease and prevention of graft rejection. Patient cells, including B cells, macrophages, DCs and even islet grafts, could be biotinylated and modified with the addition of streptavidin scaCTLA-4. All of these therapeutic approaches are aimed toward developing a system in which antigen-specific inhibition using APCs is combined with CTLA-4-mediated inhibition.

Our results demonstrate profound inhibition of antigen-specific $\mathrm{T}$ and $\mathrm{B}$ cell responses by sc $\alpha \mathrm{CTLA}-4 \mathrm{Tg}^{+} \mathrm{B}$ cells both in vitro and in vivo. Moreover, we show that selective engagement of CTLA-4 conferred significant protection against an exacerbated form of autoimmune diabetes. These results suggest that simultaneous engagement of CTLA-4 during antigen presentation may be an effective regimen for treating autoimmune diseases and organ graft rejections. To this end, we have focused on the generation of a construct that results in deliberate ligation of CTLA-4 in vivo and represents what we believe to be a novel immunotherapeutic approach. This type of antigen-specific approach may be useful for treating autoimmunity and inducing peripheral tolerance and warrants further development and consideration.

\section{Methods}

Mice. Female FVB, C57BL/6, and C3H mice were purchased from Jackson Laboratory. Female NOD mice were purchased from Taconic. NODBDC2.5 TCR $\mathrm{Tg}^{+}$mice (27) were generously provided by C. Benoist and D. Mathis (Harvard Medical School, Boston, Massachusetts, USA), and C57BL/6.CTLA-4 KO mice were generously provided by A. Sharpe (8) (Harvard Medical School, Boston, Massachusetts, USA). IFN- $\gamma$ reporter mice were generated on the C57BL/6 background and backcrossed 12 generations to NOD and subsequently bred to NOD.BDC2.5.Thy1.1 mice to generate NOD.BDC2.5.Thy1.1.Yeti mice as previously described (29). Mice were 2-8 weeks old at the initiation of the experiments. All mice were housed in a pathogen-free barrier facility at UCSF. Experiments complied with the Animal Welfare Act and NIH guidelines for the ethical care and use of animals in biomedical research and were approved by the UCSF Animal Care and Use Committee.

Abs. Monoclonal Abs to murine CD4 (RM4-5), CD8a (Ly-2), CD19 (1D3), MHC II (KH114), IgM (R6-60.2), B220 (RA3-6B2), CD40 (3/23), B7-1 (16-10A1), B7-2 (GL-1), total Ig (R26-46), IgG1 (A85-1), IgG2a (R19-15), and isotype controls were purchased from BD Biosciences - Pharmingen. Monoclonal Abs to FoxP3 (FJK-16s) were purchased from eBioscience. Purified anti-mouse IgM was purchased from Jackson ImmunoResearch Laboratories Inc.

Generation of anti-CTLA-4 scFv $\mathrm{Tg}^{+}$mice. To obtain surface-immobilized anti-CTLA-4 Abs, a single-chain, membrane-bound anti-CTLA-4 $\mathrm{Ab}(7 \mathrm{M}-4 \mathrm{~F} 10 \mathrm{scFvB} 7-1)$ was generated $(18,20)$. The cDNA encoding for $7 \mathrm{M}-4 \mathrm{~F} 10 \mathrm{scFvB}$, a mutant clone with increased affinity for CTLA-4 $(18,20)$, was cloned into Bluescript plasmid $\mathrm{P} \mu \mathrm{E} \mu$ vector, a generous gift from $\mathrm{J}$. Wilson (Princeton University, Princeton, New Jersey, USA), as previously described (21), to generate B cell expression of the ligand under the control of the IgM heavy chain enhancer and promoter. To introduce expression of anti-CTLA- 4 in mice, the DNA fragment P $\mu$ E $\mu .7 \mathrm{M}-4 \mathrm{~F} 10 \mathrm{scFv}$ B7-1 was excised using Sal I sites and microinjected into day 1 embryos from $\mathrm{FVB} / \mathrm{n}$ mice. $\mathrm{Tg}$ founders were identified by flow cytometry using mouse CTLA-4 (mCTLA-4) Ig in the presence of anti-B7-1 and anti-B7-2 Abs and backcrossed to NOD mice for 10 generations. Subsequently, the NOD.scaCTLA-4 Tg mice were crossed to NOD.B7 DKO mice.

Antigens. DNP-OVA and DNP-keyhole limpet hemocyanin (DNP$\mathrm{KLH})$ were purchased from Genemed Synthesis Inc. The amino acid composition was verified by mass spectrometry, and purity (>98\%) was assessed by HPLC.

Cell sorting. scaCTLA- $4 \mathrm{Tg}^{+} \mathrm{B}$ cells were stained with the fusion protein mCTLA-4 Ig (Genetics Institute Inc.) and with anti-B220, in the presence of anti-B7-1 and anti-B7-2 to block endogenous B7s. B220+ CTLA-4 Ig+ cells were sorted using a MoFlo cytometer high speed cell sorter (Dako). NOD.BDC2.5.Thy1.1 cells were stained with anti-CD4 FITC, anti-CD25 $\mathrm{PE}$, and anti-CD62L APC, and the $\mathrm{CD} 4^{+} \mathrm{CD} 62 \mathrm{~L}^{+} \mathrm{CD} 25^{-} \mathrm{T}$ cells were sorted. All sorted populations had greater than or equal to $98 \%$ cell purity.

Activation of donor lymphocytes, cell culture, and transfer. MoFlo-sorted WT FVB and scaCTLA-4 Tg B cells were activated by LPS $(1 \mu \mathrm{g} / \mathrm{ml}$, SigmaAldrich) in vitro for 72 hours, loaded with DNP-OVA and incubated for 4 hours at $37^{\circ} \mathrm{C}$, and transferred to naive FVB recipients. Seven days following transfer of cells, recipient animals were immunized subcutaneously in the flank with DNP-OVA (100 $\mu \mathrm{g} / \mathrm{mouse})$ in CFA (Sigma-Aldrich) containing $4 \mathrm{mg} / \mathrm{ml}$ Mycobacterium tuberculosis (BD Diagnostics).

Murine cytomegalovirus infection. NOD, NOD.scaCTLA- $4 \mathrm{Tg}^{+}$, and NOD. RAG KO mice were infected with $5 \times 10^{4} \mathrm{PFU}$ CMV Smith strain. Fourteen days after infection, liver and spleen tissue was harvested, weighed, and homogenized, and viral plaque assays were performed using NIH 3T3 mouse embryonic fibroblast cells using serial dilutions.

Assessment of insulin auto-Abs. Serum samples were collected from 5-, 10-, 20-, and 30-week-old NOD.scaCTLA-4 $\mathrm{Tg}^{+}$and NOD control mice ( $n=7-10 /$ time point/group), and insulin auto-Abs were determined as previously described (41).

Assessment of diabetes and insulitis. Blood glucose levels were measured from female NOD, NOD.scaCTLA-4 Tg' , NOD.B7 DKO, and NOD.B7 DKO. scaCTLA-4 $\mathrm{Tg}^{+}$mice weekly with an ACCU-CHEK Active glucose meter (Roche Diagnostics). Mice were considered diabetic with measurements over $250 \mathrm{mg} / \mathrm{dl}$. For histological analysis, pancreas was formalin fixed in $10 \%$ buffered formalin. Multiple $5-\mu \mathrm{m}$ sections were stained with H\&E and scored blindly for severity of insulitis as previously described (26).

CFSE adoptive transfer. To measure in vivo proliferation, $\mathrm{LN}$ cells from BDC2.5.Thy 1.1 mice were depleted of CD25+ cells using anti-CD25 (clone 7D4) and complement, followed by Ficoll density gradient centrifugation. Cells were labeled with $2.5 \mu \mathrm{M}$ CFSE (Invitrogen), and $1 \times 10^{6}$ cells were intravenously transferred to recipient mice. Recipients were sacrificed 5 days later, and CFSE dilution of pancreatic and inguinal LN cells was measured by flow cytometry using a BD LSRII cytometer (BD Biosciences - Pharmingen).

ELISAs. Assessment of cytokine production was tested for IL-2, IL-4, IL-10, and IFN- $\gamma$ by commercial ELISA kits according to manufacturer-recommended protocol (Endogen, Pierce Biotechnology). Total Ig, IgG2a, IgG1, and IgM anti-DNP Ab production was measured at days 0,7 , and 14 following transfer of antigen-loaded WT and scaCTLA- $4 \mathrm{Tg}^{+} \mathrm{B}$ cells. In brief, flatbottom microtiter plates (Nunc) were coated with DNP-KLH $(50 \mu \mathrm{g} / \mathrm{ml})$ in PBS coating buffer and blocked with $2 \%$ BSA (Sigma-Aldrich) in PBS for 1 hour at room temperature; serum samples were subsequently added in triplicate. Biotinylated anti-Ig, anti-IgG2a, anti-IgG1, and anti-IgM (BD Biosciences - Pharmingen) were added and incubated for 2 hours. Plates were developed using streptavidin-peroxidase (Zymed) and OPD substrate (Sigma-Aldrich), and absorbance was read at $405 \mathrm{~nm}$ using a Vmax kinetic microplate reader (Molecular Devices).

In vitro $T$ cell proliferation assays. Cells were cultured in 96-well microtiter plates (Corning Inc.) at $5 \times 10^{5}$ viable cells/well in complete DMEM. Cells 
were pulsed with $1 \mu \mathrm{Ci}$ of ${ }^{3} \mathrm{H}-\mathrm{TdR}$ (ICN Radiochemicals) during the last 8 hours of a 96-hour culture, and ${ }^{3} \mathrm{H}$-TdR uptake was detected using a Packard TopCount Microplate Scintillation Counter (Packard Instruments Co.).

Statistics. Statistical significance of cytokine levels, thymidine incorporation, and mean time to $25 \%$ disease incidence was analyzed using 2-tailed Student's $t$ test for comparisons of 2 means. Diabetes incidence statistical significance was analyzed using a nonparametric Wilcoxon signed-rank test. Values of $P \leq 0.05$ were considered significant.

\section{Acknowledgments}

The authors would like to thank S. Jiang and C. McArthur for technical help with cell sorting; P. Wegfahrt for mouse handling; R. Takaki and L. Lanier for expert help with the CMV plaque assays; G. Eisenbarth and L. Cheng for performing the IAA assays; and C.
Kadoch for technical assistance. We thank members of the Bluestone laboratory for critical review of this manuscript and helpful discussions. This work was supported by grants from the NIH (P01 AI035297, P30 DK063720, AI30663) and the Juvenile Diabetes Research Foundation (32004232).

Received for publication January 6, 2006, and accepted in revised form May 23, 2006.

Address correspondence to: Jeffrey A. Bluestone, Mary Margaret Clausen Distinguished Professor, UCSF Diabetes Center, University of California, San Francisco, 513 Parnassus Avenue, Box 0540, San Francisco, California 94143, USA. Phone: (415) 514-1683; Fax: (415) 564-5813; E-mail: jbluest@diabetes.ucsf.edu.
1. Walunas, T.L., et al. 1994. CTLA-4 can function as a negative regulator of $\mathrm{T}$ cell activation. Immunity. 1:405-413.

2. Walunas, T.L., Bakker, C.Y., and Bluestone, J.A. 1996. CTLA-4 ligation blocks CD28-dependent T cell activation. J. Exp. Med. 183:2541-2550.

3. Krummel, M.F., and Allison, J.P. 1995. CD28 and CTLA-4 have opposing effects on the response of $\mathrm{T}$ cells to stimulation. J. Exp. Med. 182:459-465.

4. Krummel, M.F., and Allison, J.P. 1996. CTLA-4 engagement inhibits IL-2 accumulation and cell cycle progression upon activation of resting $\mathrm{T}$ cells. J. Exp. Med. 183:2533-2540.

5. Luhder, F., Chambers, C., Allison, J.P., Benoist, C., and Mathis, D. 2000. Pinpointing when T cell costimulatory receptor CTLA-4 must be engaged to dampen diabetogenic T cells. Proc. Natl. Acad. Sci. U. S. A. 97:12204-12209.

6. Chikuma, S., Imboden, J.B., and Bluestone, J.A. 2003. Negative regulation of $\mathrm{T}$ cell receptor-lipid raft interaction by cytotoxic $\mathrm{T}$ lymphocyte-associated antigen 4. J. Exp. Med. 197:129-135.

7. Waterhouse, P., et al. 1995. Lymphoproliferative disorders with early lethality in mice deficient in Ctla-4. Science. 270:985-988.

8. Tivol, E.A., et al. 1995. Loss of CTLA-4 leads to massive lymphoproliferation and fatal multiorgan tissue destruction, revealing a critical negative regulatory role of CTLA-4. Immunity. 3:541-547.

9. Tang, Q., et al. 2004. Distinct roles of CTLA-4 and TGF-beta in CD4+CD25+ regulatory T cell function. Eur. J. Immunol. 34:2996-3005.

10. Tai, X., Cowan, M., Feigenbaum, L., and Singer, A. 2005. CD28 costimulation of developing thymocytes induces Foxp3 expression and regulatory $\mathrm{T}$ cell differentiation independently of interleukin 2. Nat. Immunol. 6:152-162.

11. Eagar, T.N., Karandikar, N.J., Bluestone, J.A., and Miller, S.D. 2002. The role of CTLA-4 in induction and maintenance of peripheral T cell tolerance. Eur. J. Immunol. 32:972-981.

12. Perez, V.L., et al. 1997. Induction of peripheral T cell tolerance in vivo requires CTLA-4 engagement. Immunity. 6:411-417.

13. Salomon, B., and Bluestone, J.A. 2001. Complexities of CD28/B7: CTLA-4 costimulatory pathways in autoimmunity and transplantation. Annu. Rev. Immunol. 19:225-252.

14. Vanderlugt, C.L., et al. 1997. Treatment with intact anti-B7-1 mAb during disease remission enhances epitope spreading and exacerbates relapses in R-EAE. J. Neuroimmunol. 79:113-118.
15. Luhder, F., Hoglund, P., Allison, J.P., Benoist, C., and Mathis, D. 1998. Cytotoxic T lymphocyte-associated antigen 4 (CTLA-4) regulates the unfolding of autoimmune diabetes. J. Exp. Med. 187:427-432.

16. Egen, J.G., and Allison, J.P. 2002. Cytotoxic T lymphocyte antigen-4 accumulation in the immunological synapse is regulated by TCR signal strength. Immunity. 16:23-35.

17. Chikuma, S., and Bluestone, J.A. 2002. CTLA-4: acting at the synapse. Mol. Interv. 2:205-208.

18. Griffin, M.D., et al. 2000. Blockade of T cell activation using a surface-linked single-chain antibody to CTLA-4 (CD152). J. Immunol. 164:4433-4442.

19. Falcone, M., Lee, J., Patstone, G., Yeung, B., and Sarvetnick, N. 1998. B lymphocytes are crucial antigen-presenting cells in the pathogenic autoimmune response to GAD65 antigen in nonobese diabetic mice. J. Immunol. 161:1163-1168.

20. Griffin, M.D., et al. 2001. Development and applications of surface-linked single chain antibodies against T-cell antigens. J. Immunol. Methods. 248:77-90.

21. Wilson, J.B., Weinberg, W., Johnson, R., Yuspa, S., and Levine, A.J. 1990. Expression of the BNLF-1 oncogene of Epstein-Barr virus in the skin of transgenic mice induces hyperplasia and aberrant expression of keratin 6. Cell. 61:1315-1327.

22. Noorchashm, H., et al. 1997. B-cells are required for the initiation of insulitis and sialitis in nonobese diabetic mice. Diabetes. 46:941-946.

23. Wong, F.S., et al. 2004. Investigation of the role of B-cells in type 1 diabetes in the NOD mouse. Diabetes. 53:2581-2587.

24. Read, S., Malmstrom, V., and Powrie, F. 2000. Cytotoxic $\mathrm{T}$ lymphocyte-associated antigen 4 plays an essential role in the function of CD25(+)CD4(+) regulatory cells that control intestinal inflammation. J. Exp. Med. 192:295-302.

25. Kingsley, C.I., Karim, M., Bushell, A.R., and Wood, K.J. 2002. CD25+CD4+ regulatory $T$ cells prevent graft rejection: CTLA-4- and IL-10-dependent immunoregulation of alloresponses. J. Immunol. 168:1080-1086.

26. Salomon, B., et al. 2000. B7/CD28 costimulation is essential for the homeostasis of the CD4+CD25+ immunoregulatory $\mathrm{T}$ cells that control autoimmune diabetes. Immunity. 12:431-440.

27. Katz, J.D., Wang, B., Haskins, K., Benoist, C., and Mathis, D. 1993. Following a diabetogenic $\mathrm{T}$ cell from genesis through pathogenesis. Cell. 74:1089-1100.

28. von Herrath, M.G., and Oldstone, M.B. 1997. Inter- feron-gamma is essential for destruction of beta cells and development of insulin-dependent diabetes mellitus. J. Exp. Med. 185:531-539.

29. Stetson, D.B., et al. 2003. Constitutive cytokine mRNAs mark natural killer (NK) and NK T cells poised for rapid effector function. J. Exp. Med. 198:1069-1076

30. Hoglund, P., et al. 1999. Initiation of autoimmune diabetes by developmentally regulated presentation of islet cell antigens in the pancreatic lymph nodes. J. Exp. Med. 189:331-339.

31. Greenwald, R.J., Freeman, G.J., and Sharpe, A.H. 2005. The B7 family revisited. Annu. Rev. Immunol. 23:515-548.

32. Greenwald, R.J., Boussiotis, V.A., Lorsbach, R.B., Abbas, A.K., and Sharpe, A.H. 2001. CTLA-4 regulates induction of anergy in vivo. Immunity. 14:145-155.

33. Hwang, K.W., et al. 2002. Cutting edge: targeted ligation of CTLA-4 in vivo by membrane-bound anti-CTLA-4 antibody prevents rejection of allogeneic cells. J. Immunol. 169:633-637.

34. Moore, A., Grimm, J., Han, B., and Santamaria, P. 2004. Tracking the recruitment of diabetogenic CD8+ T-cells to the pancreas in real time. Diabetes. 53:1459-1466.

35. Anderson, M.S., and Bluestone, J.A. 2005. The NOD mouse: a model of immune dysregulation. Annu. Rev. Immunol. 23:447-485.

36. Egen, J.G., Kuhns, M.S., and Allison, J.P. 2002. CTLA-4: new insights into its biological function and use in tumor immunotherapy. Nat. Immunol. 3:611-618.

37. You, S., et al. 2005. Autoimmune diabetes onset results from qualitative rather than quantitative age-dependent changes in pathogenic T-cells. Diabetes. 54:1415-1422.

38. Rao, S., et al. 2001. Targeted delivery of anti-CTLA-4 antibody downregulates $\mathrm{T}$ cell function in vitro and in vivo. Clin. Immunol. 101:136-145.

39. Vasu, C., Gorla, S.R., Prabhakar, B.S., and Holterman, M.J. 2003. Targeted engagement of CTLA-4 prevents autoimmune thyroiditis. Int. Immunol. 15:641-654.

40. Yolcu, E.S., Askenasy, N., Singh, N.P., Cherradi, S.E., and Shirwan, H. 2002. Cell membrane modification for rapid display of proteins as a novel means of immunomodulation: FasL-decorated cells prevent islet graft rejection. Immunity. 17:795-808.

41. Yu, L., et al. 2000. Early expression of antiinsulin autoantibodies of humans and the NOD mouse: evidence for early determination of subsequent diabetes. Proc. Natl. Acad. Sci. U. S. A. 97:1701-1706. 